\title{
BURIED INJECTOR LOGIC, A VERTICAL IIL USING DEEP ION IMPLANTATION
}

\author{
A. J. Mouthann $\dagger$ \\ Twente University of Technology, Twente, Holland
}

(Received 16 April 1986; in revised form 12 April 1987)

\begin{abstract}
A vertically integrated alternative for integrated injection logic has been realized, named buried injector logic (BIL). $1 \mathrm{MeV}$ ion implantations are used to create buried layers. The vertical pnp and npn transistors have thin base regions and exhibit a limited charge accumulation if a gate is saturated. d.c. and dynamic analysis of BIL-gate behaviour are given. A minimum gate delay of well below $1 \mathrm{~ns}$ is projected if collector areas are smaller than $10 \mu \mathrm{m}^{2}$ within an oxide isolated structure. A relation between maximum injector current density and device size is derived.
\end{abstract}

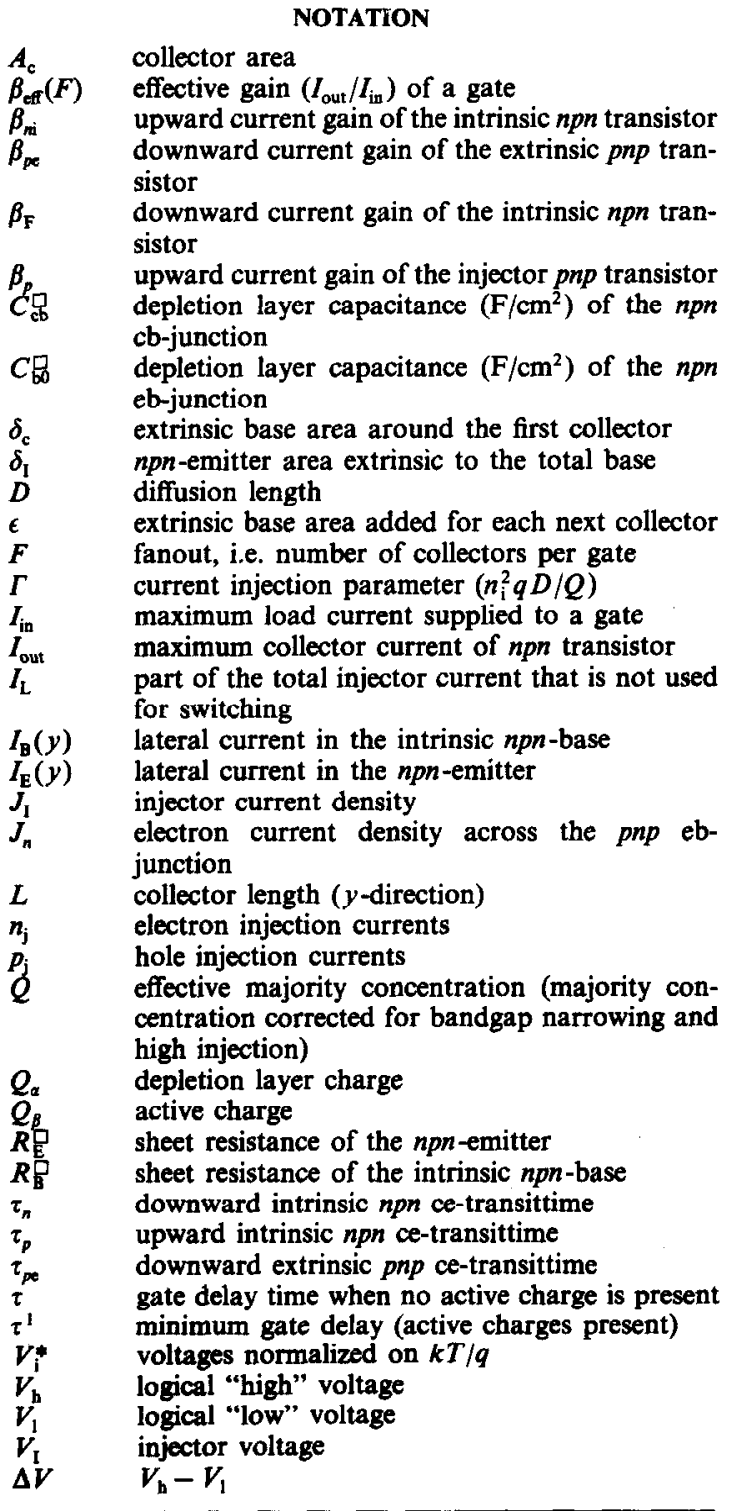

†Present address: University of Zambia, School of Engineering, Lusaka, Zambia.
$\begin{array}{ll}V_{\mathrm{B}}(y) & \text { potential in the instrinsic npn-base } \\ V_{\mathrm{E}}(y) & \text { potential in the npn-emitter }\end{array}$

\section{INTRODUCTION}

A completely vertical structure will be presented here as an alternative to the partly vertical/lateral integration of switching resp. load transistor in IIL, exhibiting a smaller minimum gate delay time. The buried layers are all ion implanted using energies up to $1 \mathrm{MeV}$.

Departing from the conventional lateral configuration of the pnp, $1 \mathrm{MeV}$ ion implantations offer the possibility to vertically stack and merge the $p n p$ and $n p n$ transistors. Figure 1 gives device structures and implantation profiles as determined with SUPREM II[1] modified with recent implantation data[2]. No diffusion is used for doping, extrinsic device areas are aiso implanted. Unwanted injection in extrinsic areas can thus be controlled. The bases of both $n p n$ and $p n p$ are thin and charge accumulation at high current levels is limited. Depletion capacitances are somewhat large due to the high doping levels causing a relatively large minimum powerdelay product.

BIL can be analysed in a similar way as Substrate Fed Logic (SFL)[3-7] and is subject to the same redefinition of effective gain of a gate compared to the effective gain for conventional IIL. Other (partly) vertical structures have been proposed in the past [8-10] showing advantages also observed for the structure proposed here.

The next two paragraphs are devoted to the d.c. and dynamic analysis of BIL, and relations are derived for the maximum fanout and minimum gate delay as function of device parameters. In the third paragraph an analysis will be given of 2-D current redistribution effects.

\section{FUNCTIONAL REQUIREMENT}

In BIL, as in SFL, the load current (i.e. the forward current of the pnp transistor) scales with the surface 


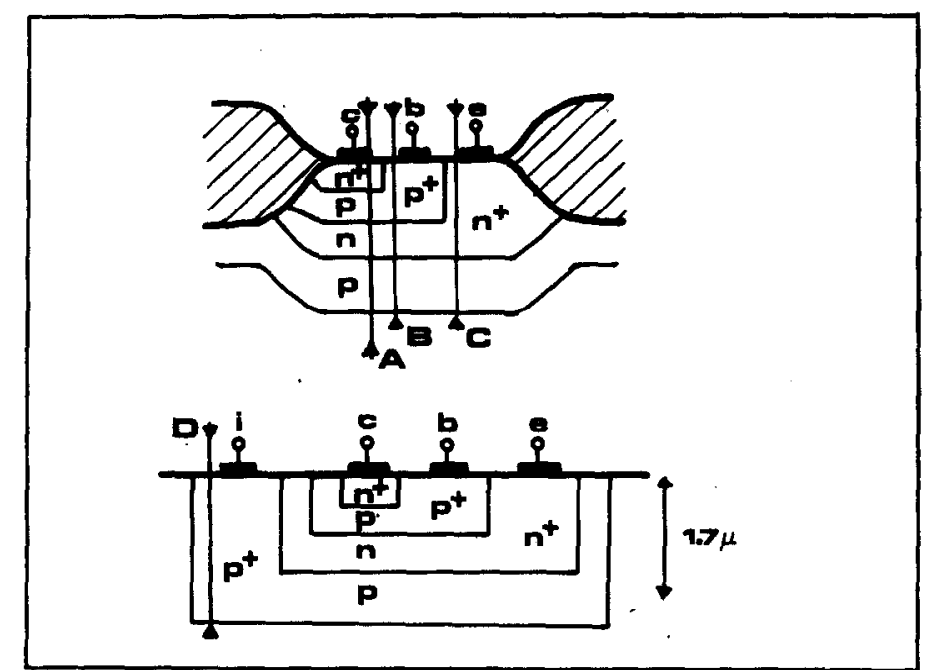

(a)

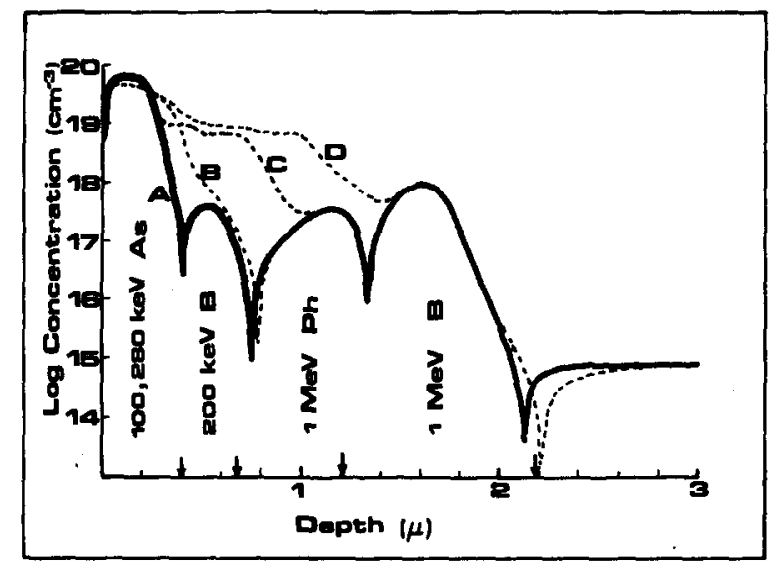

(b)

Fig. 1. (a) Realizations of BIL showing cross sections for the concentration profiles after processing in (b). Arrows indicate staining results.

area of the gate. However the gain per collector in a gate with more than one collector is independent of the number of collectors $(F)$, since the base current supply to the npn transistors also scales with the number of collectors.

The d.c. functional requirement is that the maximum load current, $I_{\text {in }}$, supplied to a particular gate must be less than the output current, $I_{\text {out }}$, that can be sunk by one collector. The ratio $I_{\text {out }} / I_{\text {in }}$ is the effective gain, $\beta_{\mathrm{eff}}(F)$, and is a function of the fanout, $F$, of the gate, and also depends on the size of the loading gate. To express $\beta_{\text {eff }}$ in terms of device parameters and device structure refer to Fig. 2. Surface areas are related to the surface area of one collector, $A_{\mathrm{C}}$ and $\delta_{c, I}$ and $c$ define extrinsic areas. These definitions also hold when LOCOS device isolation techniques are used.

In the intrinsic npn transistor we assume volume recombination negligible. Current injection parameters, $\Gamma$, are assigned to various regions in the device. Charge control relations are used to describe injection currents. For the injection current to layer a, with effective majority concentration $Q$ :

$$
I \approx A n_{\mathrm{i}}^{2} q\left(\frac{D}{Q}\right)_{\mathrm{a}} \mathrm{e}^{V / V_{T}}=A \Gamma_{\mathrm{a}} \mathrm{e}^{r^{*}}
$$

For layers with thicknesses smaller than the diffusion length, the majority concentration at low level injection is almost equal to the implanted dose. In extrinsic regions with higher concentrations and larger thicknesses $Q$ follows from integration of the dopant concentration over the diffusion length.

The load current available from a gate with fanout $F$ can be found assuming the input voltage low and consequently the $n p n$ transistor switched off:

$$
\begin{aligned}
I_{\text {in }}=p_{3}\left(F A_{\mathrm{C}}\right)+p_{4}\left[\delta_{\mathrm{c}} A_{\mathrm{C}}+(F-1) \epsilon A_{\mathrm{C}}\right] \\
=\left[\left(1+\delta_{\mathrm{c}}\right)+(1+\epsilon)(F-1)\right] A_{\mathrm{C}} \Gamma_{1} \mathrm{e}^{V_{\mathrm{i}}^{*}}
\end{aligned}
$$

with $p_{3}=p_{4}=\Gamma_{1} \mathrm{e}^{v_{\mathrm{i}}^{*}}$, and $V_{\mathrm{I}}^{*}$ the normalized injector voltage.

The current that can be sunk by one collector, with surface area $A_{\mathrm{C}}$, of an "on"-gate is:

$$
I_{\text {out }}=n_{2} A_{\mathrm{C}} \text {, }
$$




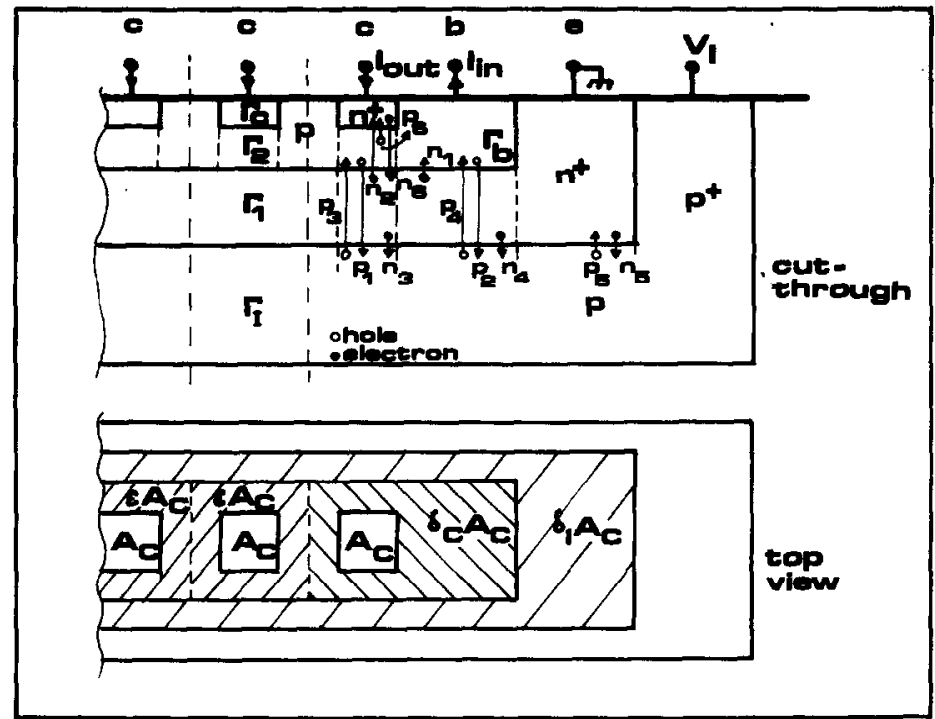

Fig. 2. Device cross-section showing hole and electron injection currents, extrinsic areas and injection parameters.

with $n_{2}=\Gamma_{2} \mathrm{e}^{V_{\mathrm{h}}^{*}}$, and $V_{\mathrm{h}}^{*}$ the normalized "high" voltage of a gate. Of course it is required that a gate that is "on" will saturate, and eqn (3) gives a maximum current. To solve the unknown $V_{\mathrm{h}}$ we consider the internal current balance of the "on"-gate when $I_{\text {in }}=0$, and the gate is not yet saturated:

$$
p_{3} A_{\mathrm{C}}+p_{4} \delta_{\mathrm{c}} A_{\mathrm{C}}=p_{1} \delta_{\mathrm{c}} A_{\mathrm{C}}+p_{2} A_{\mathrm{C}}+n_{1} \delta_{\mathrm{c}} A_{\mathrm{C}} .
$$

Evaluation leads to:

$$
\mathrm{e}^{r_{\mathrm{h}}^{*}}=\left[1+\frac{\delta_{\mathrm{c}}}{1+\delta_{\mathrm{c}}} \frac{\Gamma_{\mathrm{b}}}{\Gamma_{1}}\right]^{-1} \mathrm{e}^{r_{\mathrm{i}}}
$$

Substituting in eqn (3), and with $I_{\text {out }} / I_{\text {in }}>1$ follows:

$$
F<1+\frac{1+\delta_{c}}{1+\epsilon}\left[\frac{\beta_{n i}}{\delta_{c}+1+\frac{\delta_{c}}{\beta_{p e}}}-1\right] \text {. }
$$

The upward current gain of the intrinsic npn is $\beta_{n i}=\Gamma_{2} / \Gamma_{1}$ and is the reciprocal to the downward current gain of the intrinsic pnp. $\beta_{n i}$ can easily be varied by changing the implanted dose of the npn- base. The downward gain of the extrinsic pnp is $\beta_{p e}=\Gamma_{1} / \Gamma_{\mathrm{b}}$.

For the implantation profiles of Fig. 1(b) device simulations and measurements showed that $\beta_{n i}=8$ and $\beta_{p e}=20$. A graphical representation of eqn (5) in this case is shown in Fig. 3.

The effective gain of a gate $\beta_{\text {eff }}=I_{\text {out }} / I_{\text {in }}$ can now be evaluated as:

$$
\begin{aligned}
& \beta_{\text {eff }}(F)=\frac{I_{\text {out }}}{I_{\text {in }}}=\beta_{m i}\left[\left(\delta_{\mathrm{c}}+1\right)\right. \\
& \left.+(\epsilon+1)(F-1)+\left(1+\frac{1+\epsilon}{1+\delta_{\mathrm{c}}}(F-1)\right) \frac{\delta_{\mathrm{c}}}{\beta_{p \mathrm{c}}}\right]^{-1} .
\end{aligned}
$$

Contrary to IIL, the effective gain here is completely determined by the back injection of the pnp.

Measured d.c. characteristics of the upward $n p n$ transistor are given in Fig. 4 for a device with $F=1$, $\delta_{\mathrm{c}}=1$ and $A_{\mathrm{C}}=50 \mu \mathrm{m}^{2}$. Both $I_{\text {in }}$ (base current) and $I_{\text {out }}$ (collector current) show a deviation from the characteristic $60 \mathrm{mV}$ per current decade slope. Due to current crowding effects, which will be analysed

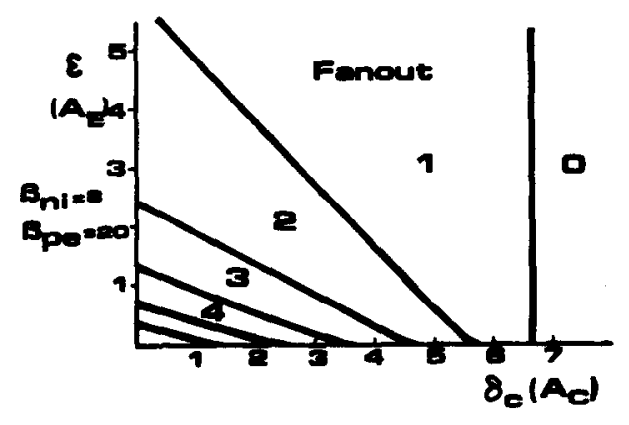

Fig. 3. Fanout as function of extrinsic device area. 


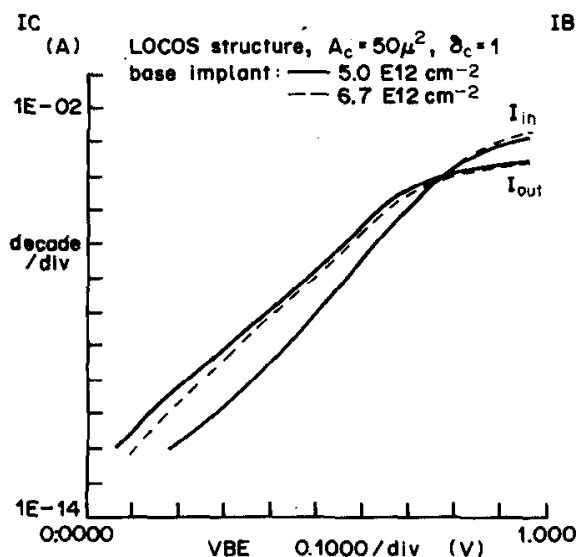

Fig. 4. d.c. Characteristics of an upward transistor. Due to current crowding, the gain reduces at higher current levels.

below, transistor gain reduces when the current reaches $10^{-4} \mathrm{~A}$. From this follows that gates can only be operated below this current range. In order to achieve high current densities small device areas are a prerequisite. In the above analysis a worst case situation is assumed: the smallest gate drives the largest. When BIL is used for random logic the above relations are safe.

\section{DYNAMIC BEHAVIOUR}

The delay time of a gate is determined here as a lumped parameter:

$$
\tau=\frac{\Sigma Q}{I},
$$

where $\Sigma Q$ is the accumulated charge in a gate, and $I$ the total current available to remove that charge. $Q$ comprises active charge $Q_{\alpha}$ and passive charge $Q_{\beta}$. Equation (6) is in fact inaccurate since not all accumulated charge is being removed at the same rate. However at higher current levels where $Q_{\alpha} \gg Q_{\beta}$ it gives a reasonable approximation because the bulk of the accumulated charge is related to the dominant current. If there is no active charge present in the device (only charged depletion capacitances) eqn (6) will only be a rough approximation.

\section{1. $Q_{a} \approx 0$}

When all gates in the logic circuit have a fanout of $F$, and we assume depletion capacitances are independent of junction voltage, it is easy to evaluate that when $Q_{\alpha} \approx 0$ :

$\tau=\frac{\left[2 F C_{\mathrm{cb}}^{\square}+\left(\left(\delta_{\mathrm{c}}+1\right)+(\epsilon+1)(F-1)\right) C_{\mathrm{b}}^{\mathrm{D}}\right] \Delta V}{\left(\beta_{p} / 1+\beta_{p}\right) J_{\mathrm{I}}\left[\left(\delta_{\mathrm{c}}+1\right)+(\epsilon+1)(F-1)\right]}$,

where $C_{\mathrm{cb}}$ and $C_{b 0}$ are then $n p n$ collector-base and base-emitter capacitances $\left(\mathrm{Fcm}^{-2}\right), \Delta V$ is the logic swing and $\beta_{p}$ the pnp upward current gain, $J_{I}$ the injector current density under the npn-base region.

\section{2. $Q_{\alpha}>Q_{\beta}$}

The active charge accumulated in the device is proportional to the current level, hence the time delay will be independent of current. Three charge components can be distinguished: charge $Q_{n p n}$ associated with the downward intrinsic npn emitter current and transit time $\tau_{n}$, charge $Q_{p n p}$ associated with the upward intrinsic npn, upward emitter current and $\tau_{p}$, and charge $Q_{p c}$ associated with the extrinsic downward $p n p, \tau_{p e}$ and extrinsic $p n p$ current. For these components we write normalized on $A_{\mathrm{C}}$ :

$$
\begin{aligned}
Q_{n p n} & =\tau_{\mathrm{n}}\left(p_{6}+n_{6}\right) F=\tau_{\mathrm{n}}\left(\Gamma_{\mathrm{c}}+\Gamma_{2}\right) F \mathrm{e}^{r_{\mathrm{h}}^{*}-V_{i}^{*}} \\
Q_{p n p} & =\tau_{\mathrm{p}}\left(p_{1}+n_{2}\right) F=\tau_{\mathrm{p}}\left(\Gamma_{1}+\Gamma_{2}\right) F \mathrm{e}^{r_{\mathrm{h}}^{*}} \\
Q_{p e} & =\tau_{p e}\left(n_{1}+p_{2}\right)\left[\delta_{\mathrm{c}}+\epsilon(F-1)\right] \\
& =\tau_{p e}\left(\Gamma_{2}+\Gamma_{1}\right)\left[\delta_{\mathrm{c}}+\epsilon(F-1)\right] \mathrm{e}^{r_{\mathrm{h}}^{*}}
\end{aligned}
$$

The transit times $\tau_{\mathrm{p}}, \tau_{\mathrm{p}}, \tau_{p e}$ can be evaluated in device simulations.

Active charge is removed by collector current drawn from the input terminal by a next gate being switched "on". The minimum available current is the collector current of a gate with fanout 1 given by eqn (3). To evaluate eqns (8) and (3) we must determine the "high" and "low" voltage from the current balance of the saturated gate. The load current to the collector equals $I_{\mathrm{in}}$. Inside the device this current is carried by $I_{\text {in }}=n_{2}-n_{6}-p_{6}$ (Fig. 2). The input terminal of the gate is "high" and carries no current, so for the base region: $\left(p_{3}+p_{4}\right)-\left(p_{1}+p_{2}\right)-p_{6}-n_{1}=0$. For a gate with fanout $F$ these relations can be rewritten:

$$
\begin{aligned}
& \left(1+\delta_{\mathrm{c}}\right) \Gamma_{1} \mathrm{e}^{\gamma_{\mathrm{t}}}=-\left(\Gamma_{2}+\Gamma_{\mathrm{c}}\right) \mathrm{e}^{V_{\mathrm{h}}^{*}-r_{i}^{*}}+\Gamma_{2} \mathrm{e}^{\gamma_{h}^{*}} \\
& {\left[\left(\delta_{\mathrm{c}}+1\right)+(\epsilon+1)(F-1)\right] \Gamma_{1}\left(\mathrm{e}^{\gamma_{\mathrm{t}}^{*}}-\mathrm{e}^{r_{\mathrm{h}}}\right)} \\
& -F \Gamma_{\mathrm{c}} \mathrm{e}^{\gamma_{\mathrm{b}}^{*}-\gamma_{\mathrm{i}}^{*}}=\left[\delta_{\mathrm{c}}+\epsilon(F-1)\right] \Gamma_{\mathrm{b}} \mathrm{e}^{\gamma_{\mathrm{h}}^{*}} .
\end{aligned}
$$

From this follows (with $\beta_{p e}, \beta_{\mathrm{F}} \gg 1$, where $\beta_{\mathrm{F}}$ is the downward current gain of the npn):

$$
\begin{aligned}
\mathrm{e}^{V_{\mathrm{h}}^{*}} & =\left[1+\frac{\beta_{n \mathrm{i}}}{\beta_{\mathrm{F}}} \cdot \frac{F}{\left(\delta_{\mathrm{c}}+1\right)+(\epsilon+1)(F-1)}\right]^{-1} \mathrm{e}^{r_{\mathrm{i}}^{*}} \\
\mathrm{e}^{V_{\mathrm{h}}^{*}-r_{\mathrm{i}}^{*}} & =\left[1-\frac{1+\delta_{\mathrm{c}}}{\beta_{n \mathrm{i}}}\right] \mathrm{e}^{r_{\mathrm{h}}^{*}}
\end{aligned}
$$

Substituting eqn (10) into eqns (8) and (3) yields for eqn (6) (again $\beta_{F}, \beta_{p e} \gg 1$ ):

$$
\begin{aligned}
& \tau^{1}=F\left(1-\frac{1+\delta_{c}}{\beta_{n i}}\right) \tau_{n}+F\left(1+\frac{1}{\beta_{n i}}\right) \tau_{p} \\
& +\left(\delta_{\mathrm{c}}+\epsilon(F-1)\right)\left(1+\frac{1}{\beta_{n i}}\right) \tau_{p e}
\end{aligned}
$$

Equation (11) shows that the larger $\beta_{m i}$, the closer $\tau^{1}$ will be equal to the sum of $n p n$-downward, $n p n$ upward (or pnp-downward) and extrinsic pnpdownward collector-emitter transit times. For smaller $\beta_{n \mathrm{i}} \tau^{1}$ will increase since generally $\tau_{n}<\tau_{p}, \tau_{p e}$. For the profile of Fig. 1(b) transit times at an injector current 


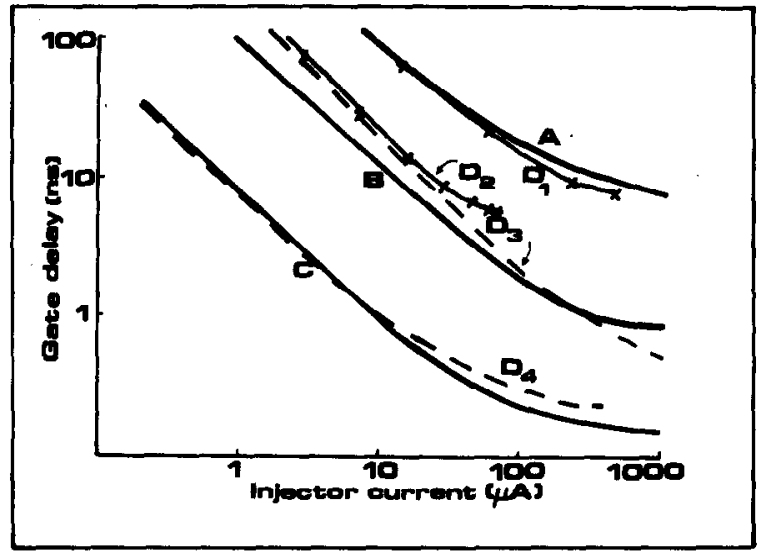

Fig. 5. Gate delay as function of injector current. A, B, C conventional structures with oxide isolation. A: $1.2 \mu \mathrm{m}$ epi-layer, $5 \mu \mathrm{m}$ design rules[11], B: $0.85 \mu \mathrm{m}$ epi-layer, $2.5 \mu \mathrm{m}$ design rules[12], C: $0.2 \mu \mathrm{m}$ epi-layer, $0.5 \mu \mathrm{m}$ design rules $\left(7 \mu \mathrm{m}^{2}\right.$ cell area)[11]. $\mathrm{D}$ : BIL structures ( $\mathrm{x}$, measurements). $\mathrm{D}_{1}: 300 \mu \mathrm{m}^{2}$ collector area, $\delta_{\mathrm{c}}=2$, with junction isolation showing high depletion capacitances. $D_{2}: 50 \mu \mathrm{m}^{2}$ collector area, $\delta_{c}=1$ oxide isolation, $5 \mu \mathrm{m}$ technology showing measurements and $1-D$ theory $\left(\mathrm{D}_{3}\right)$. Note the discrepancy between actual 3-D behaviour and the 1-D theory. $D_{4}$ : projected delay of a cell of $7 \mu \mathrm{m}^{2}$.

density of $1000 \mathrm{~A} / \mathrm{cm}^{2}$ were, using device simulations, found to be $\tau_{n}=4 \times 10^{-11}, \tau_{p}=8 \times 10^{-11}$ and $\tau_{p e}=10^{-10} \mathrm{~s}$. Equation (11) can be combined with eqn (7) to give gate delay as function of injector current density. Curves $D_{1}, D_{2}, D_{3}, D_{4}$ in Fig. 5 show the results and measurements on ring counters with a fanout of 1 , compared with optimized conventional IIL delay times. A more detailed analysis of the measurements follows in the next paragraph.

The minimum power-delay product follows from $p d=V_{\mathrm{I}} I_{\mathrm{L}}+0.5 \Sigma C \Delta V^{2}$, where $I_{\mathrm{L}}$ accounts for the d.c. losses. For a gate with $F=1$ using eqn (8) with $\Delta V=V_{1}$ (neglecting $p_{5}$ ):

$$
\begin{aligned}
& C_{\mathrm{cb}} \rightarrow C_{\mathrm{cb}}^{\mathrm{a}} \\
& C_{\mathrm{b} 0} \rightarrow C_{\mathrm{b} 0}^{\square} \\
& \text { and } \\
& \mathrm{pd}=\left(\frac{\delta_{\mathrm{l}}+\delta_{\mathrm{c}}+1}{\beta_{\rho}\left(\delta_{\mathrm{c}}+1\right)}+\frac{1}{2}\right)\left(2 C_{\mathrm{cb}}+\left(\delta_{\mathrm{c}}+1\right) C_{\mathrm{b} 0}\right) A_{\mathrm{C}} V_{\mathrm{I}}^{2}
\end{aligned}
$$

For a collector area of $50 \mu \mathrm{m}^{2}, \delta_{1}=0$ and $\delta_{\mathrm{c}}=1$ the $\mathrm{pd}=0.08 \mathrm{pJ}$. The value found in ringcounter measurements is $0.1 \mathrm{pJ}\left(\mathrm{D}_{2}\right.$ in Fig. 5). Sidewall capacitances however are not incorporated here, and should not be neglected.

\section{2-D CURRENT CROWDING EFFECTS}

The intrinsic npn base has a sheet resistance of several $k \Omega / \square$, depending on the implanted dose. d.c. Simulations of a gate in the off-state, using a distributed 2-D circuit model as in Fig. 6(a), show that for a LOCOS isolated device with a collector area of $50 \mu \mathrm{m}^{2}, \delta_{\mathrm{c}}=1$, a vertical current density of $10^{3} \mathrm{~A} / \mathrm{cm}^{2}$ causes a detrimental lateral potential drop in the intrinsic $n p n$-base with $3.5 \mathrm{k} \Omega / \square$. Due to this potential drop locally the eb-junction of the npn remains forward biased, leaving the gate partly on, thereby at least increasing the "low" voltage. In Figure 6(b) the current density across each junction is plotted as function of position for a gate in the on- and in the off-state. When the device is scaled down linearly with a factor 2 the gate behaves properly in the off-state.

An analytical relation between the maximum injector current density and device size can be derived for the case that the eb-junction of the upward $n p n$ will not carry a forward current. We assume here only lateral current flow in the $y$-direction, c.f. Fig. 6(a). The base current, $I_{\mathrm{B}}(y)$; is transported in the negative $y$-direction, starting at $y=L$. The emitter current, $I_{E}(y)$ flows in the positive $y$-direction for $y>0$ and in the negative for $y<0$, assuming symmetry. An expression relating $V_{\mathrm{B}}(L)$ to injector current density and device size is now derived, taking into account a lateral potential drop in the npn-emitter. In a similar way as in Ref. [8] an expression for $I_{E}(y)$ can be found using the boundary conditions $V_{\mathrm{E}}(L)=0$ and $I_{\mathrm{E}}(0)=0$ :

$$
I_{\mathrm{B}}(y)=\frac{2 L V_{\mathrm{T}}}{R_{\mathrm{E}}^{\square}} B \tan B y,
$$

with $B$ a constant to be evaluated from:

$$
\frac{B}{\cos B L}=\left(\frac{J_{n} R_{\mathrm{E}}}{2 V_{\mathrm{T}}}\right)^{1 / 2}
$$

Here $J_{n}$ is the electron current density across the eb-junction of the injector pnp near $y=L$, directly following from the injector voltage.

From equation (13) follows:

$$
V_{\mathrm{E}}(y)=-2 V_{\mathrm{T}} \ln \frac{\cos B y}{\cos B L}
$$

Since $I_{B}(y)$ is the pnp collector current, we can write:

$$
\frac{\mathrm{d} I_{\mathrm{B}}(y)}{\mathrm{d} y}=L J_{n} \mathrm{e}^{-V_{\mathrm{E}}(y) / V_{\mathrm{T}}}
$$

Also:

$$
V_{\mathrm{B}}(y)=-\frac{R_{\mathrm{B}}^{\square}}{L} \int_{0}^{y} I_{\mathrm{B}}(y) \mathrm{d} y .
$$




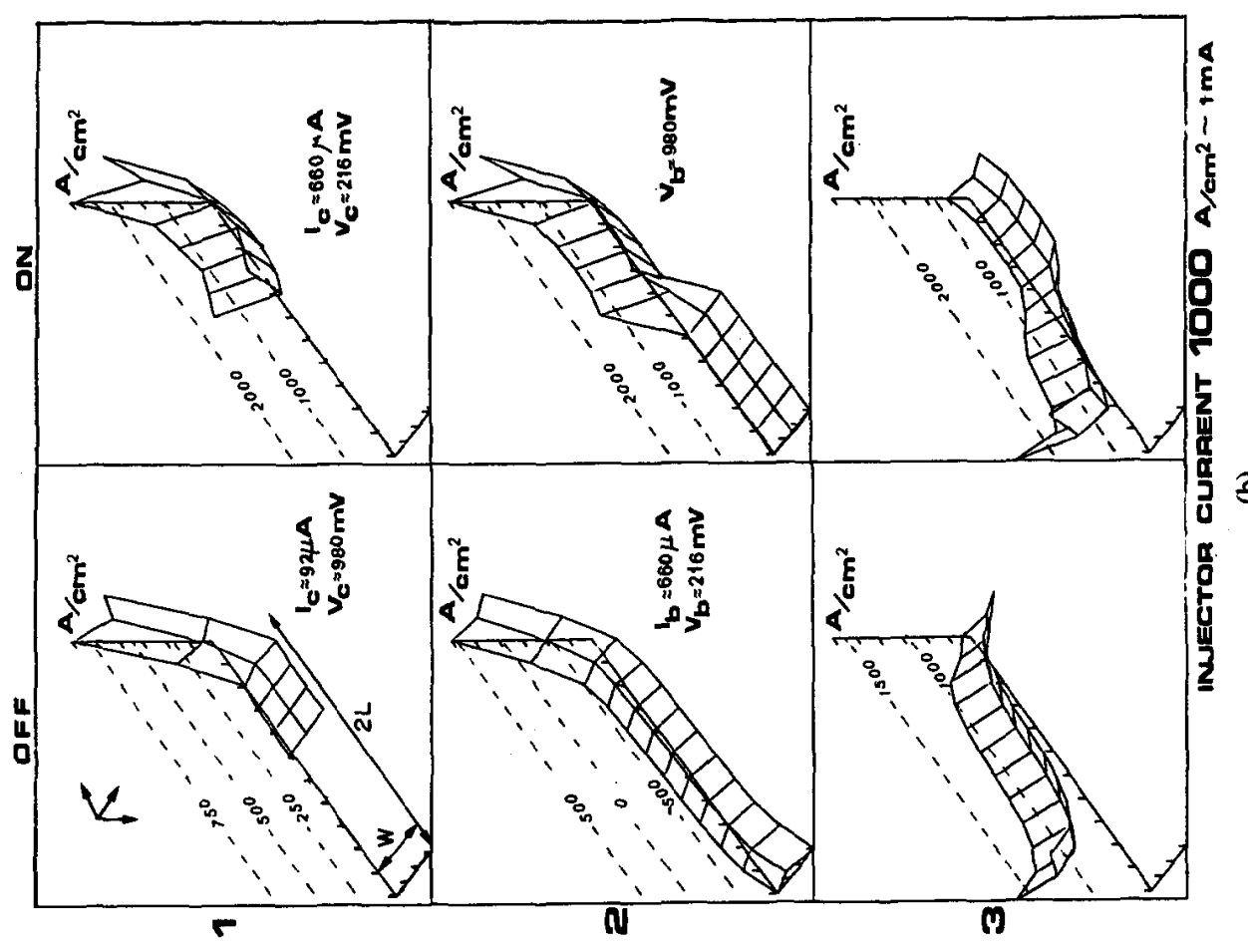

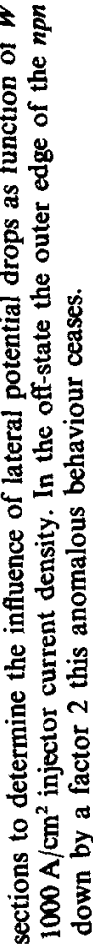

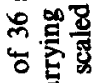

도.

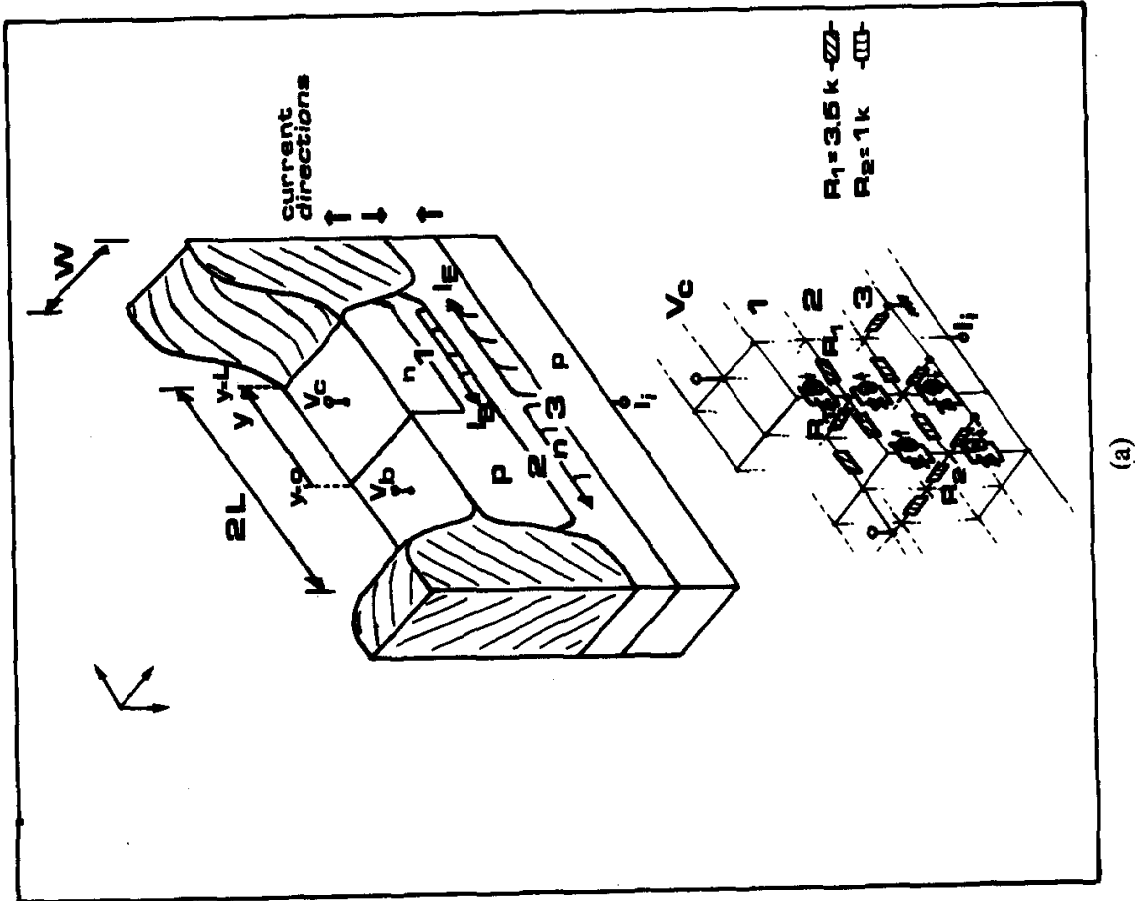

.

空

돈

㖊焉

ำ 웜

형.

of

ठ

露息

동.s 톤

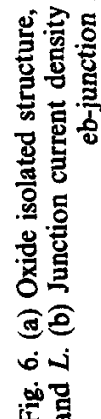




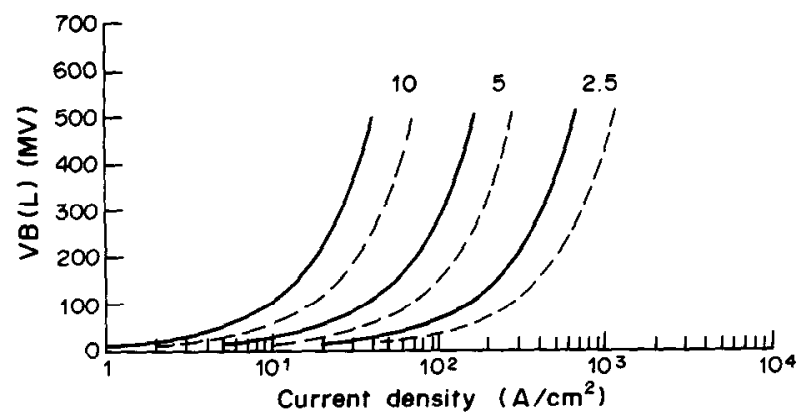

Fig. 7. $V_{\mathrm{B}}(L)$ as function of $J_{n}$ for devices with $L=10,5$ and $2.5 \mu \mathrm{m}$. $R_{\mathrm{E}}^{\mathrm{Q}}=1 \mathrm{k} \Omega / \square$, solid line $R_{B}=6.5 \mathrm{k} \Omega / \square$, dashed line $R_{B}=3.5 \mathrm{k} \Omega / \square$.

Solving equation (16) and substituting with eqn (15) in eqn (17) yields:

$$
V_{\mathrm{B}}(y)=\frac{R_{\mathrm{B}}^{\square} J_{n}}{B^{2} \cos \mathrm{BL}}(\cos B y-B y \sin B L-1) \text {. }
$$

The voltage $V_{\mathrm{B}}(L)$ is plotted as function of $J_{n}$ in Fig. 7 , where $L$ and $R_{B}$ are parameters.

Comparing the results of Fig. 7 with the measurements $D_{2}$ in Fig. 2 shows reasonable agreement. In Figure 2 the maximum injector current density was $100 \mathrm{~A} / \mathrm{cm}^{2}$, while from Fig. 7 a maximum $J_{n}$ of $50 \mathrm{~A} / \mathrm{cm}^{2}$ follows. The injector current density follows from $\left(\beta_{p}+1\right) J_{n}$. In actual devices however $I_{E}$ is not restricted to the $y$-direction only.

\section{CONCLUSIONS}

Deep ion implantation has shown to be an interesting technique for the realization of a fully vertical version of IIL, here named Buried Injector Logic. Both $n p n$ and $p n p$ transistors have small transit times projecting the minimum gate delay well below $1 \mathrm{~ns}$ if collector areas are in the order of $10 \mu \mathrm{m}^{2}$ or below $\left(R_{\mathrm{B}}=3.5 \mathrm{k} \Omega / \square\right)$. The usual advantages of vertical IIL as lay-out facilitation, absence of powerlines at the silicon surface (in the case when $p$-wafers are used) are also present. Since ion implantation is the only source of doping high device reproducability is likely, and a fine tuning is possible to optimize device characteristics. No extensive diffusion steps are necessary, simplifying processing. Because of its projected high speed the application of BIL in memory circuits with BIL flip-flops or cross-coupled thyristor cells is attractive and requires no extra masking.

Acknowledgements - The author wishes to thank A. A. I. Aarnink for the assistance given in device processing and Professor J. Middelhoek for the fruitful discussions we had on the subject. B. H. Essink is thanked for his assistance in designing test structures. These investigations in the program of the Foundation for Fundamental Research on Matter (FOM) have been supported by the Foundation for Technical Research (STW), future technical science branch of the Netherlands Organization for the Advancement of Pure Research (ZWO).

\section{REFERENCES}

1. D. A. Antoniadis, S. E. Hansen and R. W. Dutton, Stanford El. Lab. Rep. no 5019-2 (1978).

2. S. Oosterhoff, Ph.D. Thesis, Twente University of Technology, Holland (1986).

3. P. S. Walsh, V. Blatt and L. W. Kennedy, ESSCIRC 1975, p. 122 (1975).

4. V. Blatt, P. S. Walsh and L. W. Kennedy, IEEE $J$. Solid-St. Circ. SC-10, 336 (1975).

5. P. S. Walsh and G. W. Sumerling, IEEE J. Solid-St. Circ. SC-12, 123 (1977).

6. V. Blatt and G. W. Sumerling, IEEE J. Solid-St. Circ. SC-12, 128 (1977).

7. G. Perlegus and Shu-Park Chan, IEEE J. Solid-St. Circ. SC-13, 491 (1978).

8. O. Tomisawa, Y. Horiba, S. Kato, K. Murakami, A. Yasuoka and T. Nakamo, IEEE J. Solid-St. Circ. SC-11, 637 (1976).

9. W. P. Dumke, IBM Tech. Discl. Bull. 21, 4242 (1979).

10. A. A. Yiannoulos, ISSCC 1978, p. 12 (1978).

11. S. A. Evans, IEEE J. Solid-St. Circ. SC-14, 318 (1979).

12. D. D. Tang, T. H. Ning, R. D. Isaac, G. C. Feth, S. K. Wiedmann and $H$. Nien-Yu, IEEE Trans. Electron Dev. ED-27, 1379 (1980). 